\title{
Employing the therapeutic operating characteristic (TOC) graph for individualised dose prescription
}

\author{
Aswin L Hoffmann ${ }^{1,2^{*}}$, Henk Huizenga ${ }^{1}$ and Johannes HAM Kaanders ${ }^{1}$
}

\begin{abstract}
Background: In current practice, patients scheduled for radiotherapy are treated according to 'rigid' protocols with predefined dose prescriptions that do not consider risk-taking preferences of individuals. The therapeutic operating characteristic (TOC) graph is applied as a decision-aid to assess the trade-off between treatment benefit and morbidity to facilitate dose prescription customisation.

Methods: Historical dose-response data from prostate cancer patient cohorts treated with 3D-conformal radiotherapy is used to construct TOC graphs. Next, intensity-modulated (IMRT) plans are generated by optimisation based on dosimetric criteria and dose-response relationships. TOC graphs are constructed for dose-scaling of the optimised IMRT plan and individualised dose prescription. The area under the TOC curve (AUC) is estimated to measure the therapeutic power of these plans.

Results: On a continuous scale, the TOC graph directly visualises treatment benefit and morbidity risk of physicians' or patients' choices for dose (de-)escalation. The trade-off between these probabilities facilitates the selection of an individualised dose prescription. TOC graphs show broader therapeutic window and higher AUCs with increasing target dose heterogeneity.

Conclusions: The TOC graph gives patients and physicians access to a decision-aid and read-out of the trade-off between treatment benefit and morbidity risks for individualised dose prescription customisation over a continuous range of dose levels.
\end{abstract}

Keywords: Radiotherapy, Treatment planning, Individualisation, Dose-response relations, Decision-making

\section{Background}

The main task in radiation dose prescription and treatment planning is to maximise the tumour control probability (TCP) while maintaining an acceptable normal tissue complication probability (NTCP). Currently, this compromise is 'frozen' in treatment protocols, which are based on consensus opinions about what is considered to be the best trade-off for a specific patient population. However, in an era where patient empowerment enters into clinical practice, subjective criteria reflecting the physician's or individual patient's risk-taking preferences

\footnotetext{
* Correspondence: aswin.hoffmann@maastro.nl

'Department of Radiation Oncology, Radboud University Nijmegen Medical Center, P.O. Box 9101, Nijmegen 6500 HB, The Netherlands

${ }^{2}$ Department of Radiation Oncology (MAASTRO), GROW School for Oncology and Developmental Biology, Maastricht University Medical Center, P.O. Box 1588, Maastricht 6201 BN, The Netherlands
}

should inherently be taken into account to establish a 'customised' treatment. Therefore, individualised treatment prescription and planning requires decision-making based on TCP and NTCP scores rather than on dosimetric criteria alone.

Amols et al. proposed an a posteriori decision-making approach to rank existing treatment plans having different combinations of TCP and NTCP based on a single figure of merit quantifying the physician's preferences [1]. Another approach is to extract patient preferences prior to treatment. In a recent prospective trial involving patients with localised prostate carcinoma scheduled for threedimensional conformal radiotherapy (3D-CRT), patients were offered an a priori choice between treatments with two alternative dose levels resulting in different probabilities for tumour control and side-effects [2,3]. From this study, it

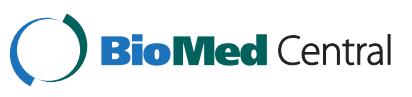


became clear that the majority (79\%) of patients opted to be involved in the choice of their treatment once a decision-aid was provided, and most patients attached a higher weight to quality-of-life related aspects than to tumour control [4].

The availability of modern treatment techniques provides the ability to design a variety of treatment plans with divergent trade-offs between benefit and morbidity. The ultimate way to take this variety into account is to predict TCP and NTCP scores for the individual patient based on the 3D dose distribution and fractionation scheme applied. This is challenging, since the clinical outcome measures for these treatments are not well known. Furthermore, specific tumour characteristics determining radiation response (e.g., intrinsic radiosensitivity and hypoxia) are often unknown for the individual patient. Nevertheless, various models and parameter sets to estimate TCP and NTCP from a 3D dose distribution have been developed and applied (e.g., [5-8]). It is generally believed that these models are adequate to rank rival treatment plans provided they are used over the dose range from which they have been derived. Some of these models have been incorporated into modern treatment planning systems, enabling a 'radiobiological' evaluation of treatment plans. However, the TCP/NTCP trade-off is difficult to assess in current treatment planning systems, even for a simple 'dose scalarisation' approach where only the prescription dose of a given treatment plan is changed for either a fixed number of fractions or for a fixed fraction dose. The concept of maximizing the probability of uncomplicated tumour control, $P_{+}$, as a function of dose has been proposed to find the single optimum dose level for this approach $[9,10]$. The criticism against this measure is that an a priori 'rigid' trade-off between TCP and NTCP is assumed without knowing their interrelationship over the range of potential dose prescriptions. As such, it is not suitable as a single measure for dose prescription customisation.

The aim of the present paper is to apply the concept of the therapeutic operating characteristic (TOC) graph to assess the trade-off in the TCP/NTCP domain over a continuous range of prescribed dose levels for given treatment plans. The TOC graph is presented as an interactive tool for dose prescription customisation of a treatment plan for an individual patient. We compare the $P_{+}$and TOC graph and discuss their value for individualised dose prescription optimisation. The concept is illustrated by a clinical example of prostate cancer where the trade-off between 5-year biochemical no evidence of disease $\left(\mathrm{bNED}_{5}\right)$, late gastrointestinal (GI) and genitourinary (GU) morbidity is studied.

\section{Methods}

Therapeutic operating characteristic (TOC) graph

The TOC is a parametric plot of TCP vs. NTCP with the prescribed dose as a continuous independent parameter
[11-15]. As TCP and NTCP increase with total dose, their interrelationship presents an ascending curve in the benefit-injury decision space. The TOC graph can be used to estimate the optimal level of therapeutic effect and the associated dose level, based on individual risktaking preferences.

So far, few quantitative measures of therapeutic window or therapeutic power have been published. Both refer to a quality index for radiotherapy to achieve loco-regional tumour control and to prevent severe late side effects. We propose to use the area under the curve (AUC) of the TOC graph as an index of the therapeutic power of a treatment technique or plan, independent of consensus on the prescribed dose level. This is by analogy with the AUC of the ROC graph used in diagnostic radiology [16].

\section{TOC graph for a patient population: modelling results from clinical studies}

The TOC graph was first applied to clinical outcome data from a systematic literature review on the effects of radiation dose on tumour control and morbidity in the treatment of prostate cancer [3]. The trade-off between TCP (i.e., bNED b , $\mathrm{NTCP}_{\mathrm{GI}}$ and $\mathrm{NTCP}_{\mathrm{GU}}$ (late GI and GU morbidity Grade $\geq 2$ RTOG) was assessed for three-dimensional conformal radiotherapy (3D-CRT) techniques of combined data from dose escalation studies. The models and parameters used to describe the dose-effect relationship for the patient population studied are summarised in Additional file 1.

\section{TOC graph for an individual patient: technique assessment and dose prescription customisation}

TCP and NTCP models derived from the literature were applied to a 3D dose distribution of a given initial treatment plan to construct TOC graphs by a posteriori variation of the total prescription dose. This was either accomplished by variation of the number of fractions (at constant dose-per-fraction assuming no tumour cell repopulation correction for overall treatment time) or by variation of the fraction dose (while keeping the number of fractions constant). TOC graphs were generated after treatment planning to allow for TCP/NTCP balancing and selection of the preferred prescription for therapy delivery. Taking the individual's risk-taking preferences into account, a treatment plan with a customised dose prescription can be selected as a point on the TOC graph. We illustrate the concept of dose-level scaling for a typical prostate cancer patient using different treatment delivery and plan optimisation techniques in a step-wise approach. Firstly, TCP/NTCP evaluation was done for a forward planned 3D-CRT dose distribution that had initially been designed for standard fractionation. Secondly, a TOC graph was generated for an intensitymodulated radiotherapy (IMRT) plan obtained by inverse 
planning using dose and dose-volume (i.e., physical) objectives with equivalent initial prescribed dose (IMRT $\mathrm{Thys}_{\text {phs }}$ ), as it was expected that the NTCP of the IMRT $_{\text {phys }}$ plan was lower under tumour iso-effective conditions than for the 3D-CRT plan. Then a third plan (IMRT $\mathrm{I}_{\text {biol }}$ ) was obtained from the IMRT $\mathrm{T}_{\text {phys }}$ plan by physico-biological optimisation, improving the TCP under isotoxic conditions. The hypothetical benefit of scaling the IMRT $\mathrm{I}_{\text {biol }}$ was also assessed. TOC graphs and AUCs of the three plans were compared.

\section{Organ segmentation and standard treatment plans}

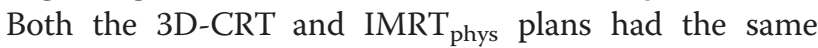
dose prescription recipe: 78 Gy in 39 fractions satisfying the $95 \%$ and $107 \%$ under- and overdosage criteria according to ICRU 50 criteria. The planning target volume (PTV) encompassed the prostate gland and base of the seminal vesicles plus a $5 \mathrm{~mm}$ isotropic margin. The rectum and bladder were delineated as organs at risk (OARs). The 3D-CRT plan comprised a wedged $10 \mathrm{MV}$ co-planar 4-beam arrangement. The IMRT $\mathrm{T}_{\text {phys }}$ plan encompassed a $10 \mathrm{MV}$ co-planar 5-beam geometry and was generated for a maximum of 60 step-and-shoot segments by inverse treatment planning (Pinnacle ${ }^{3}$ version 7.6c; Philips Radiation Oncology Systems, Fitchburg, USA) with direct machine parameter optimisation (DMPO; RaySearch Laboratories AB, Stockholm, Sweden).

\section{Physico-biological treatment plan optimisation}

For isotoxic optimisation, values for $\mathrm{NTCP}_{\mathrm{GI}}$ and NTCP $_{G U}$ were calculated from the IMRT $T_{\text {phys }}$ plan, and constituted upper limits for the IMRT $_{\text {biol }}$ plan at fixed fraction number $(N=39)$. The physical objectives from the IMRT $_{\text {phys }}$ plan were converted into constraints, resulting in the following physico-biological optimisation problem:

$$
\begin{array}{ll}
\text { maximise } & \operatorname{TCP}(\underline{D}) \\
\text { subject to } & \operatorname{NTCP}_{\mathrm{GI}}(\underline{D}) \leq n t c p_{\mathrm{GI}} \\
& \operatorname{NTCP}_{\mathrm{GU}}(\underline{D}) \leq n t c p_{\mathrm{GU}} \\
& \operatorname{DVH}_{i}\left(D, V_{j}\right) \leq d v h_{i, j}
\end{array}
$$

where $\underline{D}$ is the dose distribution to be optimised, and $n t c p_{\mathrm{GI}}, \bar{n} t c p_{\mathrm{GU}}, d v h_{\mathrm{i}, \mathrm{j}}$ are constraint values for organ $i$ and dose-volume constraint $j$ as obtained from the IMRT $_{\text {phys }}$ plan after it had been generated by inverse planning. Nonclinical research software (ORBIT Workstation, version 1.5; RaySearch Laboratories AB, Stockholm, Sweden) was used to solve this problem with DMPO [17]. The dose-response models used are summarised in Additional file 2.

For TOC analysis, treatment plans were retrieved from Pinnacle $^{3}$ and ORBIT Workstation into an in-house developed software tool (MATLAB version 7.6.0; The MathWorks Inc., Natick, USA). For the tumour a conservative $(\alpha / \beta)_{\mathrm{T}}=2$ Gy adopted from [18] was applied, while for both OAR endpoints generally accepted values of $(\alpha / \beta)_{\mathrm{OAR}}=3$ Gy and 6 Gy were adopted for Grade $\geq 2$ late GI [19] and late GU toxicity [20], respectively. These $(\alpha / \beta)$ ratios were used to account for voxel-based fractionation correction prior to calculating TCP and NTCP scores.

\section{Results}

\section{TOC graph for patient population}

Figure 1 illustrates the population-averaged dose-response graphs for TCP and NTCP as a function of the prescribed dose level in the 2 Gy equivalent dose $\left(E Q D_{2}\right)$ range of 60-80 Gy, obtained from a systematic literature review [3].

In Figure 2A, two TOC graphs depict the trade-offs between the TCP, $\mathrm{NTCP}_{\mathrm{GI}}$, and $\mathrm{NTCP}_{\mathrm{GU}}$ of Figure 1 when plotted against each other. Figure $2 \mathrm{~B}$ illustrates the TOC graph zoomed in on the $\mathrm{EQD}_{2}$ range of $60-80$ Gy for GI morbidity. In this graph, the dose level for which the increase in TCP and NTCP with dose is equal, and hence $P_{+}=T C P-N T C P$ achieves its maximum value, is 72 Gy with associated $\mathrm{TCP}=75 \%$ and $\mathrm{NTCP}=$ $12 \%$. Below this level, the gain in TCP per unit dose is larger than the increase in NTCP, whereas the converse is true beyond this level.

In Figure 3, the interdependence between TCP, NTCP and $\mathrm{NTCP}_{\mathrm{GU}}$ as a function of the prescribed dose is shown in a $3 \mathrm{D}$ TOC graph together with its $2 \mathrm{D}$ projections on the

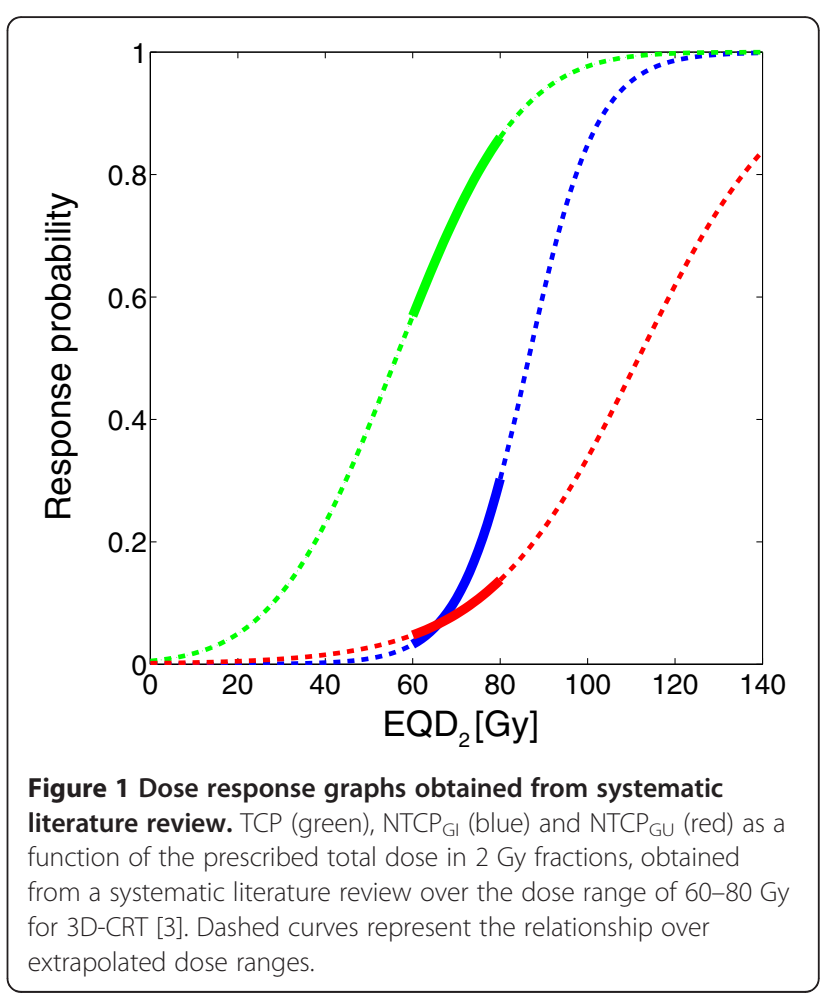



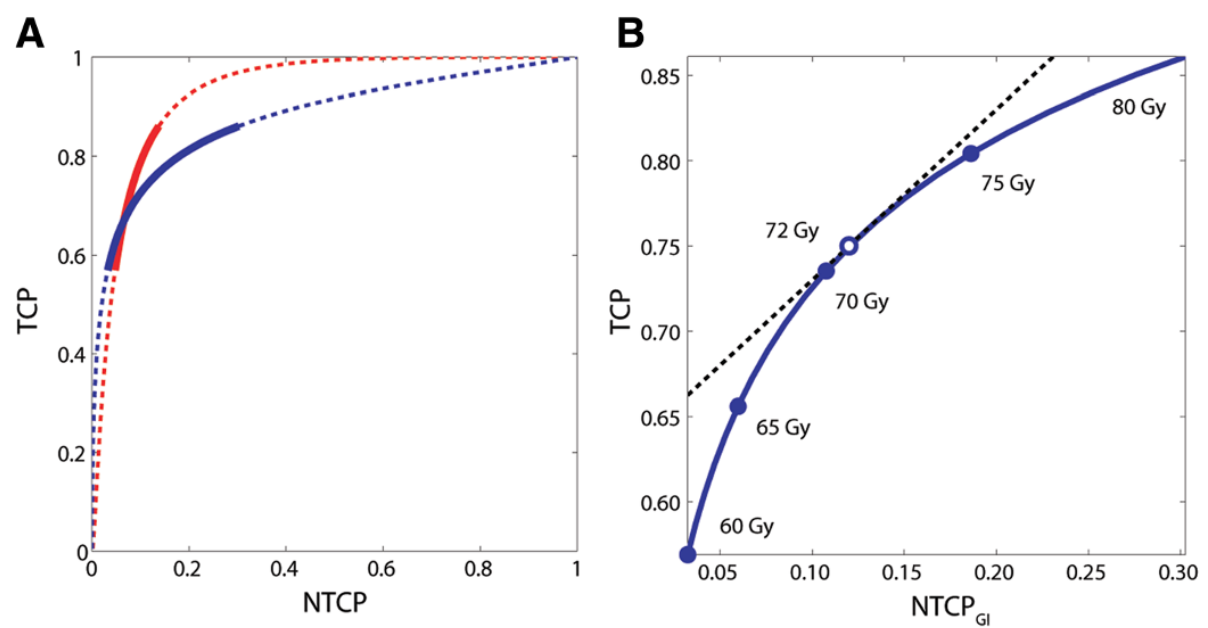

Figure $\mathbf{2}$ TOC graphs reconstructed from systematic literature review. TOC graphs showing (A) the trade-offs between TCP and either NTCP (blue) or NTCP $\mathrm{GU}_{\mathrm{G}}$ (red) over the dose range of 60-80 Gy in 2 Gy fractions (solid curve) and the extrapolated dose ranges (dashed curve). In (B) the dashed line represents the tangent where TCP and NTCP $P_{G}$ equally increase with dose and defines the optimum of $P_{+}=T C P-N T C P(O)$. Dose levels in $2 \mathrm{~Gy}$ fractions are indicated $(\bullet)$ along the curve.

$\mathrm{TCP} / \mathrm{NTCP}_{\mathrm{GI}}$ and $\mathrm{TCP} / \mathrm{NTCP}_{\mathrm{GU}}$ space that is shown in Figure 2A.

\section{TOC graphs for individual patient}

In Figures $4 \mathrm{~A}$ and $4 \mathrm{~B}$, TOC graphs are shown for the initial (i.e., $39 \times 2$ Gy) 3D-CRT treatment plan $(\mathrm{TCP}=83 \%$, $\mathrm{NTCP}=25 \%)$ as a function of the scaled dose per fraction and the number of fractions, respectively. Dose statistics for renormalised plans are given in Table 1. It is evident that both curves coincide at the nominal dose prescription of 2 Gy per fraction or $N=39$ fractions. Their relative position in the TCP/NTCP space is insensitive to the model parameters $\left(\mathrm{TD}_{50}, \gamma_{37}, m, a\right)$, and only depends on the ratio of $(\alpha / \beta)_{\mathrm{T}}$ to $(\alpha / \beta)_{\mathrm{OAR}}$. Since $(\alpha / \beta)_{\mathrm{T}}<(\alpha / \beta)_{\mathrm{OAR}}$, escalating the total dose beyond the original 78 Gy level by adding fractions will increase the TCP/NTCP ratio less than by increasing the fraction dose. This becomes apparent from Table 1 when comparing the TCP and NTCP scores for the plans with a $5 \%$ nominally higher prescription dose of $82 \mathrm{~Gy}$, obtained either from scaling the fraction size to 2.1

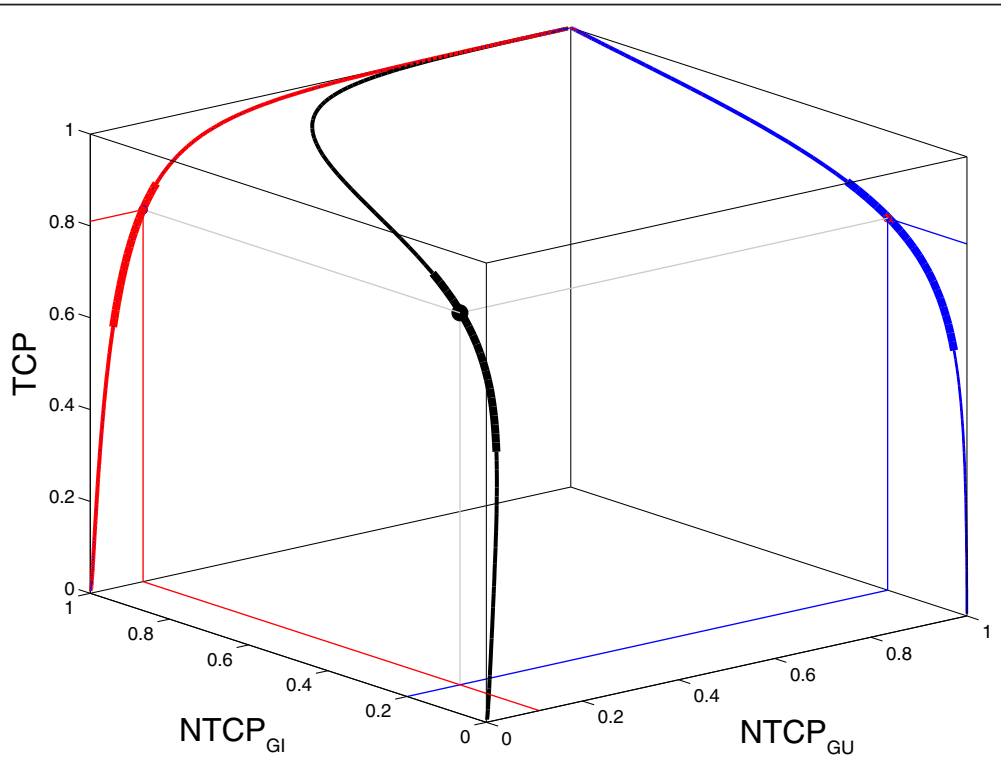

Figure 3 3D TOC graph reconstructed from systematic literature review. Multidimensional TOC graph (black) for TCP, NTCP ${ }_{G l}$, and NTCP as a function of the prescribed dose in 2 Gy fractions. Projection of TOC graphs for TCP vs. NTCP GU (red) and TCP vs. NTCP (blue) are shown. Thin curves represent the relationship over extrapolated dose ranges; the 78 Gy plan is indicated $(\bullet)$. 

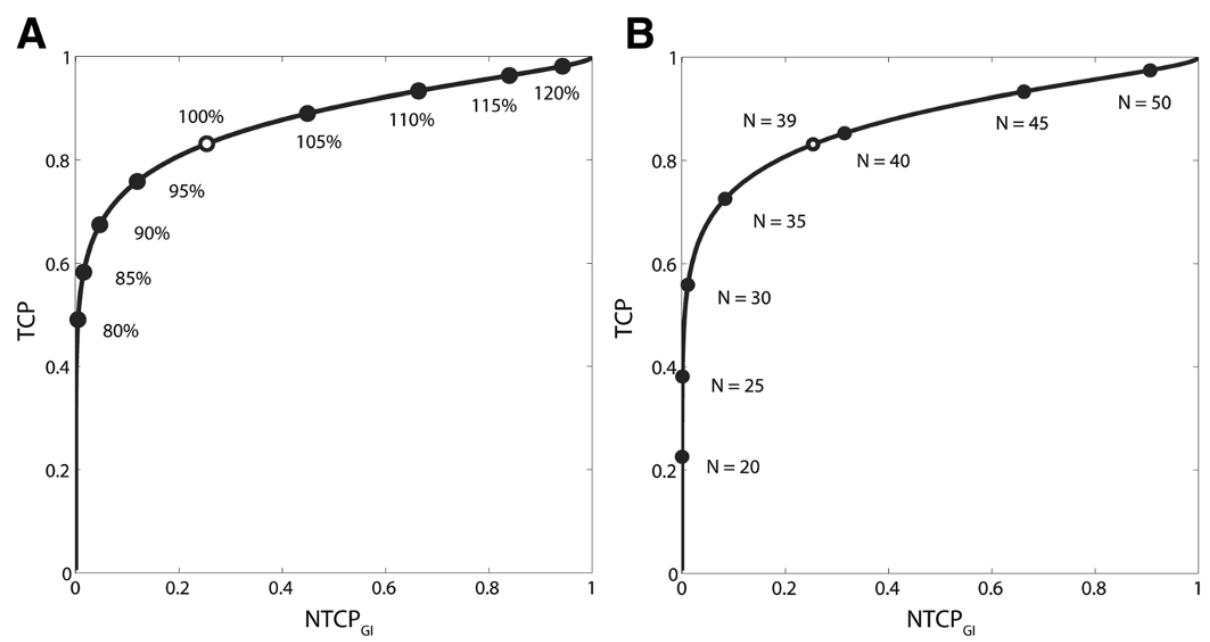

Figure 4 TOC graphs for fraction size and fraction number variation. TOC graphs for prostate 3D-CRT plan showing trade-off between TCP and $\mathrm{NTCP}_{\mathrm{Gl}}$ as a function of $(\mathbf{A})$ the dose-per-fraction scale factor (at constant fraction number) and (B) the number of fractions $N$ (at constant fraction dose). The initial plan ( $39 \times 2 \mathrm{~Gy})$ is marked (O) at the $100 \%$ dose level and at $\mathrm{N}=39$, respectively.

Gy (with 39 fractions) or from increasing the number of fractions to 41 (with 2 Gy per fraction). The opposite holds in case of dose de-escalation below 78 Gy and for $(\alpha / \beta)_{\mathrm{T}}>(\alpha / \beta)_{\text {OAR }}$. When plotted in the same graph, both TOC curves would coincide only if $(\alpha / \beta)_{\mathrm{T}}=(\alpha / \beta)_{\mathrm{OAR}}$.

In Figure 5, TOC graphs for the 3D-CRT, IMRT $\mathrm{T}_{\text {phys }}$, and $\mathrm{IMRT}_{\text {biol }}$ plans for the same patient anatomy are shown. Dose statistics for the initial plans are given in Table 2. By comparing the TOC graphs for the 3D-CRT and the IMRT plans, it is obvious that the IMRT $\mathrm{I}_{\text {phys }}$ plan has a more conformal dose distribution than the 3D-CRT plan, reducing NTCP (from $25 \%$ to $13 \%$ ) at constant TCP $=83 \%$. Comparison of the TOC graphs for the IMRT phys $_{\text {. }}$ and the $\mathrm{IMRT}_{\text {biol }}$ plan suggests that an increase in TCP (from $83 \%$ to $87 \%$ ) can be obtained at constant $\mathrm{NTCP}=13 \%$. It can be seen that the three TOC graphs do not cross and have different AUCs (3D-CRT: 0.87, IMRT $_{\text {phys: }}: 0.91$, IMRT $\left._{\text {biol }}: 0.93\right)$. The IMRT $_{\text {biol }}$ plan outperforms the IMRT $\mathrm{I}_{\text {phys }}$ plan over the whole range of prescribed dose levels and fractionation schemes, whereas the latter outperforms the 3D-CRT plan. By comparing the TOC graphs, its use for prescription dose customisation becomes apparent; when rescaling a given treatment plan does not fulfill the TCP/NTCP trade-off requirements, only re-optimization (with more direct steering of the TCP/ NTCP criteria) will improve the quality of the plan.

Table 1 Dose and response statistics for renormalised 3D-CRT plans

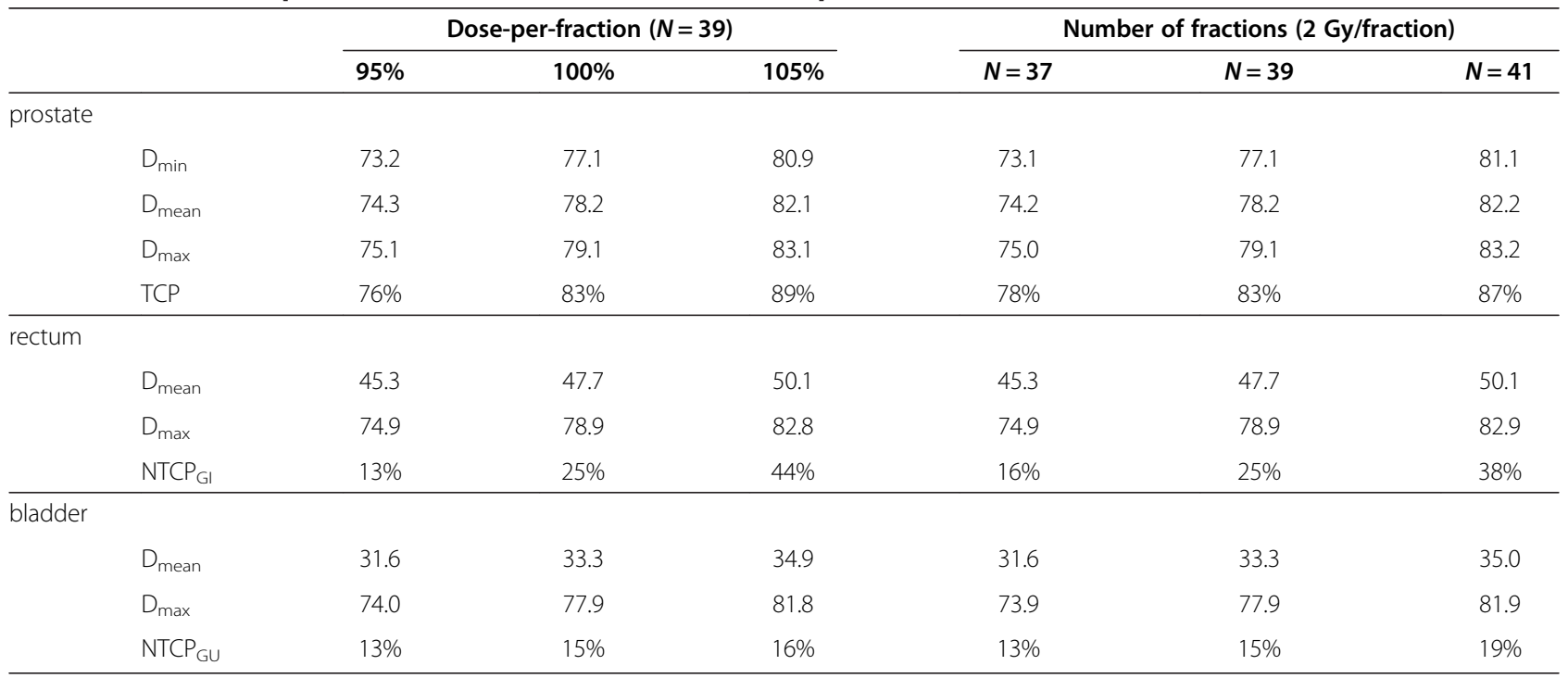

Abbreviations: $N$, number of fractions; $D_{\min }$, minimum dose; $D_{\text {mean }}$ mean dose; $D_{\text {max }}$ maximum dose, $T C P$, tumour control probability, $N T C P_{G \prime}$ probability of late gastrointestinal morbidity; $N T C P_{G U}$, probability of late genitourinary morbidity. 


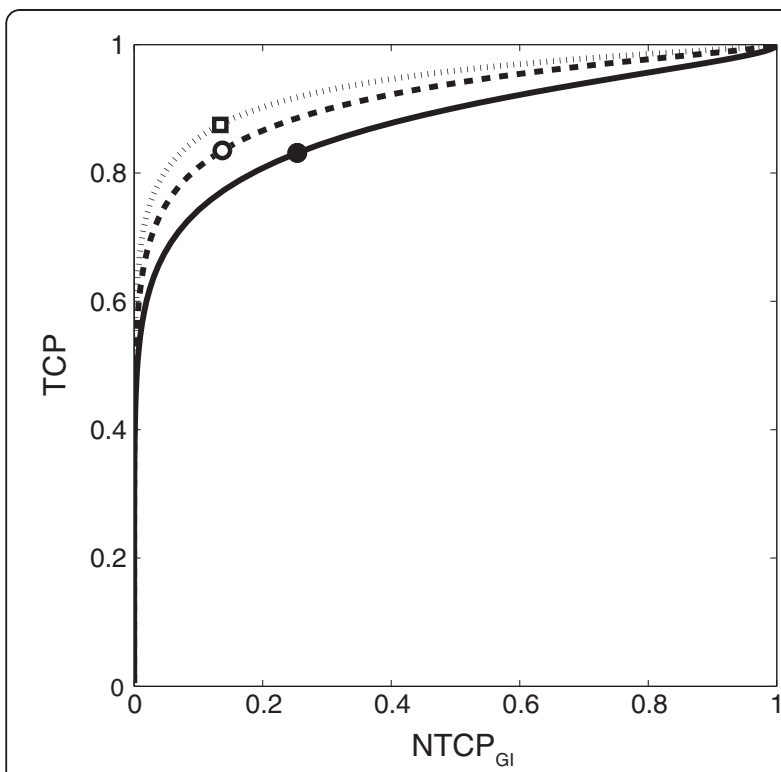

Figure 5 TOC graphs for 3D-CRT, IMRT phys $_{\text {and IMRT }}$ biol plans. TOC graphs for TCP vs. NTCP $\mathrm{Gl}$ obtained by dose level scaling of a prostate 3D-CRT plan (solid curve), the IMRT phys plan (dashed curve), and the IMRT biol plan (dotted curve) at constant fraction number, $N=39$. Symbols $(\bullet, O, \square)$ represent the TCP and NTCP scores of the initial plans.

Figure 5 clearly shows that both the TOC's shape and its AUC help to estimate the therapeutic power of a treatment plan over a continuous range of TCP and NTCP scores. Finally, the TOC graph of the best performing $\left(\mathrm{IMRT}_{\text {biol }}\right)$ plan can be presented to the

Table 2 Dose and response statistics for initial treatment plans (39 fractions)

\begin{tabular}{|c|c|c|c|c|}
\hline & & 3D-CRT & IMRT $_{\text {phys }}$ & IMRT $_{\text {biol }}$ \\
\hline \multicolumn{5}{|c|}{ prostate } \\
\hline & $D_{\min }$ & 77.1 & 75.4 & 74.1 \\
\hline & $\mathrm{D}_{\text {mean }}$ & 78.2 & 78.8 & 81.8 \\
\hline & $\mathrm{D}_{\max }$ & 79.1 & 81.8 & 97.0 \\
\hline & $\mathrm{TCP}$ & $83 \%$ & $83 \%$ & $87 \%$ \\
\hline \multicolumn{5}{|c|}{ rectum } \\
\hline & $\mathrm{D}_{\text {mean }}$ & 47.7 & 36.8 & 36.3 \\
\hline & $\mathrm{D}_{\max }$ & 78.9 & 80.1 & 84.7 \\
\hline & $\mathrm{NTCP}_{\mathrm{Gl}}$ & $25 \%$ & $13 \%$ & $13 \%$ \\
\hline \multicolumn{5}{|c|}{ bladder } \\
\hline & $\mathrm{D}_{\text {mean }}$ & 33.3 & 25.1 & 17.7 \\
\hline & $\mathrm{D}_{\max }$ & 77.9 & 82.4 & 93.8 \\
\hline & $\mathrm{NTCP}_{\mathrm{GU}}$ & $15 \%$ & $14 \%$ & $14 \%$ \\
\hline
\end{tabular}

Abbreviations: 3D-CRT, three-dimensional conformal radiation therapy; IMRT phys, physically optimised IMRT plan; IMRT biol, physico-biologically optimised IMRT plan; $D_{\text {min }}$, minimum dose; $D_{\text {mean }}$ mean dose; $D_{\text {max }}$ maximum dose, $T C P$, tumour control probability, $N T C P_{G l,}$ probability of late gastrointestinal morbidity; $N T C P_{G U}$, probability of late genitourinary morbidity. physician and/or patient to assess the trade-off between treatment benefit and morbidity and to choose from the TOC graph the dose-level that best fits their preferences.

\section{Discussion}

In an era of individualised cancer therapy, radiotherapy should move towards customised dose prescription in order to maximise individual patient's outcome and quality of life. The currently applied 'rigid' treatment protocols neither take into account the anatomical diversity of patients within the risk group nor their individual risk-taking preferences. The standard strategy will lead to relative underdosage in individuals who are willing to tolerate a higher radiation dose aiming for tumour control, whilst relatively overdosing those not willing to accept the possible adverse effects resulting from the predefined radiation dose. Hence, there is a need to move toward customised treatment planning where individualisation is not restricted to adapting the spatial dose distribution to the patient's anatomy, but also involves balancing of treatment benefit and morbidity in terms of TCP and NTCP.

As an overture towards individualised TCP and NTCP risk balancing, we conducted a prospective decisionmaking trial in patients with localised prostate carcinoma scheduled for 3D-CRT, who were offered an a priori restricted choice between treatments with two alternative pre-selected dose levels [2]. To take this one step further, a tool to depict a continuum of clinically relevant dose levels and their corresponding TCP and NTCP indices would be helpful to assist selecting an optimum dose level after an initial treatment plan has been generated for the individual patient.

In previous work by Lind et al., the concept of maximizing the probability of uncomplicated tumour control, $P_{+}$, as a function of dose has been proposed to find the optimum dose level [10]. It should be noted that the TOC graph provides unbiased information in comparison to the $P_{+}$graph when plotted as a function of dose. This becomes clear when the general expression for $P_{+}$is considered:

$$
P_{+}=(T C P-N T C P+\delta\{N T C P(1-T C P)\}),
$$

where $\delta$ is the estimated fraction of patients for which tumour and normal tissue response are statistically independent $(0 \leq \delta \leq 1)$ [9]. Expression (1) assumes that an implicit a priori trade-off between TCP and NTCP is made. For example, with $\delta=0$ equation (1) reduces to $P_{+}=T C P-N T C P$, assuming that the risk of recurrence is equally important as the risk of suffering from (severe) side effects. Therefore, $P_{+}$has often been criticised as a measure that does not reflect the clinical reality that reductions in TCP are rated differently from the risk of complications by clinicians and patients. By plotting TCP 
vs. NTCP with the prescribed dose as an independent parameter, no assumption with regard to $a$ priori risktaking preferences between TCP and NTCP is made. Instead, their interrelationship is assessed over the whole range of potential dose levels to facilitate the selection of an optimum that satisfies the risk-taking preferences of the individual clinician or patient. Using the full TOC graph instead of selecting the single dose level where $P_{+}$is maximal pre-empts the criticism against $a$ priori balancing the TCP/NTCP trade-off.

In the 1970's Moore and Mendelsohn proposed the TOC curve as a method to optimise treatment levels in cancer therapy [11] and later on, it was used in studies on radiation therapy for head and neck tumours [12-14]. However, this concept has only been employed to determine an optimum dose level for a patient population in a 'one size fits all' approach. We emphasize that with the current possibilities and knowledge of inverse treatment planning techniques (e.g., by explicitly incorporating dose-response relationships into the optimization as objective and/or constraint functions) the application options for individualized dose prescription strategies may be more important and clinically relevant than before.

The TOC graph used in the current work provides a means to visualise and explore the trade-off between TCP and NTCP in an intuitive manner to be used as a tool for a posteriori dose prescription customisation of an initial treatment plan for an individual patient. Plans with different TCP/NTCP trade-offs can be generated from the same underlying relative dose distribution by scaling of the prescribed dose or fractionation scheme. Since no re-planning is required, the TOC graph can be generated off-line. Furthermore, this approach facilitates the interactive balance between TCP and NTCP of a given plan after treatment planning and plan optimisation and does not require risk-taking predilections to be articulated a priori, as is the case in today's inverse treatment planning approaches. Additionally, by exploiting the AUC, a quantitative definition of the therapeutic power is provided independent of consensus on the dose level.

The clinical application of changing the prescribed dose and fractionation after an initial treatment plan has been generated has recently gained renewed interest as part of individualised dose prescription strategies that escalate the tumour dose until maximally tolerable NTCP limits are reached in, for example, non-small cell lung cancer radiotherapy $[6,7,21,22]$. Current treatment planning systems lack the means to assess the effects of dose or fractionation variation of a given treatment plan in terms of TCP and NTCP indices and do not provide insight in their interrelationship. Consequently, it is common practice to completely re-design and recalculate a treatment plan once the dose description or fractionation schedule has changed. Our re-scaling approach together with the TOC concept brings individualised dose prescription into clinical practice by providing an intuitive and easy-to-apply tool to find the preferred prescription dose for either a fixed number of fractions (by changing the dose per fraction) of for fixed fraction dose (by changing the number of fractions) which yields pre-selected NTCP limit(s) for the OAR(s).

\section{Practical use of TOC graph}

To exploit our concept in the clinical workflow, first, a TOC graph is generated from historical dose-response data that were derived for a group of patients treated with the same irradiation technique but with different dose prescriptions. The individual patient and the radiation oncologist interactively choose TCP/NTCP coordinates from the TOC graph in a first decision-making step, which results in an intended dose prescription. Subsequently, an initial treatment plan is designed for the intended dose prescription based on the individual patient's anatomy. Hence, the population-based TOC can be used to establish an evidence-based 'customised' dose prescription for a given treatment technique prior to designing a treatment plan for the intended dose prescription level. Finally, a second decision-making step is required to further customise the dose prescription by re-normalisation and to achieve at least the desired, but likely superior, TCP/NTCP scores.

\section{Physico-biological treatment plan optimisation}

We showed that constrained optimisation in inverse planning based on TCP and NTCP models resulted in an iso-toxic treatment plan with improved TCP. As the TCP model allows for some degree of dose heterogeneity in the PTV, a dose reduction in parts of the PTV is exploited to reduce the NTCP, whereas in other parts the dose is escalated. A steep dose fall-off at the border of the PTV keeps the TCP constant, and allows for better sparing of the OAR, which facilitates dose escalation resulting in the same NTCP with higher TCP.

\section{Relation of TOC graph to Pareto efficient frontier}

The TOC graph should not be confused with the Pareto efficient frontier (PEF) that has been discussed in radiotherapy lately (e.g., [23]). The principles of TOC and PEF are fundamentally different. Whereas the TOC graph has been obtained from scaling a single treatment plan, a PEF would have required the physico-biological optimisation problem to be repeatedly solved to optimality for different a priori set NTCP constraint values. A comparison between the TOC and PEF is however beyond the scope of this paper and will be addressed in another paper by the authors.

Due to uncertainties in the radiobiological models, the choice for a final dose level should not solely be based on the TOC graph, but should always include the underlying 
3D dose distribution. Preferably, the TOC graph should be presented together with the model parameters, $\mathrm{TCP} /$ NTCP values, and confidence levels [13].

However, confidence intervals for all model parameters are not available or may be unreliable for IMRT dose distributions, as they have mainly been derived from 3D conformal radiotherapy dose distributions. As more clinical data comes available the TOC-based estimate of the optimal dose level will get more trustworthy. We therefore recommend to carefully introduce the TOC into clinical practice, by gradually releasing the interval of dose-level re-scaling towards values where the uncertainties are largest. The true value of the TOC graph and underlying TCP/NTCP models should be assessed in clinical trials comparing the predicted and actual TCP and NTCP values.

\section{Conclusions}

For an individual patient, the TOC graph can be exploited as an a posteriori decision aid in risk-adapted dose prescription customisation of a given treatment plan as a function of the prescribed dose level or the number of fractions. It provides physicians and patients with a decision aid for individual risk-taking preferences in terms of TCP/NTCP trade-off. The AUC is a dose level independent measure of the therapeutic power of a treatment plan.

\section{Additional files}

Additional file 1: TCP and NTCP models used in systematic literature review.

Additional file 2: TCP and NTCP models used by ORBIT Workstation.

\begin{abstract}
Abbreviations
AUC: Area under the curve; bNED 5 : 5-year genitourinary biochemical no evidence of disease; DVH: Dose-volume histogram; EQD 2 : Equivalent dose in 2 Gy fractions; Gl: Gastrointestinal; GU: Genitourinary; ICRU: International commission on radiation units and measurements; IMRT: Intensity-modulated radiation therapy; IMRT phys: Physically optimised IMRT plan; IMRT biol: Physicobiologically optimised IMRT plan; MV: Megavolt; NTCP: Normal tissue complication probability; OAR: Organ at risk; $P_{+}$: Uncomplicated tumour control probability; PEF: Pareto efficient frontier; PTV: Planning target volume; ROC: Receiver operating characteristic; RTOG: Radiation therapy oncology group; TCP: Tumour control probability; TOC: Therapeutic operating characteristic; 3D-CRT: Three-dimensional conformal radiation therapy.
\end{abstract}

\section{Competing interests}

All authors declare to have no competing interests.

\section{Authors' contributions}

ALH, conceived and designed the study, carried out the data collection, performed the data analysis and interpretation, and drafted the manuscript. $\mathrm{HH}$, participated in the design of the study and the interpretation of the data, and helped to draft the manuscript. JHK, contributed substantially to the critical revision of the paper. All authors read and approved the final manuscript.

\section{Acknowledgements}

We thank Julia van Tol-Geerdink for providing the data of the systematic literature review on the effects of radiation dose on tumour control and morbidity in the treatment of prostate cancer [3]. Fredrik Carlsson and Kjell Eriksson from RaySearch Laboratories AB (Stockholm, Sweden) are acknowledged for their help with the ORBIT Workstation research software.

Received: 10 December 2012 Accepted: 28 February 2013

Published: 7 March 2013

\section{References}

1. Amols HI, Zaider M, Heyes MK, Schiff PB: Physician/patient-driven risk assignment in radiation oncology: reality or fancy? Int J Radiat Oncol Biol Phys 1997, 38:455-461

2. Van Tol-Geerdink JJ, Stalmeier PF, Van Lin EN, Schimmel EC, Huizenga H, Van Daal WA, Leer JW: Do prostate cancer patients want to choose their own radiation treatment. Int J Radiat Oncol Biol Phys 2006, 66:1105-1111.

3. Van Tol-Geerdink JJ, Stalmeier PFM, Pasker-de Jong PCM, Huizenga H, Van Lin EN, Schimmel EC, Leer JW, Van Daal WA: Systematic review of the effect of radiation dose on tumour control and morbidity in the treatment of prostate cancer by 3D-CRT. Int J Radiat Oncol Biol Phys 2006, 64:534-543.

4. Van Tol-Geerdink JJ, Stalmeier PF, Van Lin EN, Schimmel EC, Huizenga H, Van Daal WA, Leer JW: Do patients with localised prostate cancer treatment really want more aggressive treatment. J Clin Oncol 2006, 24:4581-4586

5. Warkentin B, Stavrev P, Stvreva N, Field C, Fallone BG: A TCP-NTCP estimation module using DVHs and known radiobiological models and parameter sets. J App/ Clin Med Phys 2004, 5:50-63.

6. Van Baardwijk A, Bosmans G, Bentzen S, Boersma L, Dekker A, Wanders R, Wouters BG, Lambin P, De Ruysscher D: Radiation dose prescription for non-small-cell lung cancer according to normal tissue dose constraints: an in silico clinical trial. Int J Radiat Oncol Biol Phys 2008, 71:1103-1110.

7. Hoffmann AL, Troost EG, Huizenga H, Kaanders JH, Bussink J: Individualized dose prescription for hypofractionation in advanced non-smal-cell lung cancer radiotherapy: an in silico trial. Int J Radiat Oncol Biol Phys 2012, 83:1596-1602

8. Van Baardwijk A, Wanders S, Boersma L, Borger J, Öllers M, Dingemans AM, Bootsma G, Geraedts W, Pitz C, Lunde R, Lambin P, De Ruysscher D: Mature results of an individualized radiation dose prescription study based on normal tissue constraints in stage I to III non-small-cell lung cancer. J Clin Oncol 2010, 28:1380-1386.

9. Brahme A: Treatment optimization using physical and radiobiological objective functions. In Radiation Therapy Physics. Edited by Smith AR. Berlin: Springer; 1995:209-246.

10. Lind BK, Mavroidis P, Hyödynmaa S, Kappas C: Optimisation of the dose level for a given treatment plan to maximise the complication-free tumour cure. Acta Oncol 1999, 38:787-798.

11. Moore DH, Mendelsohn ML: Optimal treatment levels in cancer therapy. Cancer 1972, 30:97-106.

12. Tokars RP, Griem ML: Carcinoma of the nasopharynx and optimisation of radiotherapeutic management for tumour control and spinal cord injury. Int J Radiat Oncol Biol Phys 1979, 5:1741-1748.

13. Metz CE, Tokars RP, Kronman HB, Griem ML: Maximum likelihood estimation of dose-response parameters for therapeutic operating characteristic (TOC) analysis of carcinoma of the nasopharynx. Int J Radiat Oncol Biol Phys 1982, 8:1185-1192.

14. Ågren A, Brahme A, Turesson I: Optimisation of uncomplicated control for head and neck tumours. Int J Radiat Oncol Biol Phys 1990, 19:1077-1085.

15. Andrews JR: Benefit, risk and optimisation by ROC analysis in cancer therapy. Int J Radiat Oncol Biol Phys 1985, 11:1557-1562.

16. Hanley JA, McNeil BJ: The meaning and use of the area under a receiver operating characteristic (ROC) curve. Radiology 1982, 143:29-36.

17. Eriksson $\mathrm{K}$, Rehbinder $\mathrm{H}$ : Advanced tools for radiobiological evaluation and optimisation of treatment plans. In Proceedings of XVth International Conference on the Use of Computers in Radiation Therapy (ICCR). Volume II: 4-7 June 2007. Toronto: University of Toronto; 2007:60-64.

18. Miralbell R, Roberts SA, Zubizarreta E, Hendry JH: Dose-fractionation sensitivity of prostate cancer deduced from radiotherapy outcomes of 5,969 patients in seven international institutional datasets: $\alpha / \beta=1.4$ (0.9 2.2) Gy. Int J Radiat Oncol Biol Phys 2012, 82:e17-e24 
19. Michalski JM, Gay H, Jackson A, Tucker SL, Deasy JO: Radiation dose-volume effects in radiation-induced rectal injury. Int J Radiat Oncol Biol Phys 2010, 76(Suppl 3):S123-S129.

20. Dale E, Hellebust TP, Skjønsberg A, Høgberg T, Olsen DR: Modeling normal tissue complication probability from repetitive computed tomography scans during fractionated high-dose-rate brachytherapy and external beam radiotherapy of the uterine cervix. Int J Radiat Oncol Biol Phys 2000, 47:963-971.

21. Uzan J, Nahum AE: Radiobiologically guided optimisation of the prescription dose and fractionation scheme in radiotherapy using BioSuite. Br J Radiol 2012, 85:1279-1286.

22. Vinogradskiy Y, Tucker SL, Bluett JB, Wages CA, Liao Z, Martel MK: Prescribing radiation dose to lung cancer patients based on personalized toxicity estimates. J Thorac Oncol 2012, 7:1676-1682.

23. Ottosson RO, Engstrom PE, Sjöstrom D, Behrens CF, Karlsson A, Knöös T, Ceberg C: The feasibility of using Pareto fronts for comparison of treatment planning systems and delivery techniques. Acta Oncol 2009, 48:233-237.

doi:10.1186/1748-717X-8-55

Cite this article as: Hoffmann et al.: Employing the therapeutic operating characteristic (TOC) graph for individualised dose prescription. Radiation Oncology 2013 8:55.

\section{Submit your next manuscript to BioMed Central and take full advantage of:}

- Convenient online submission

- Thorough peer review

- No space constraints or color figure charges

- Immediate publication on acceptance

- Inclusion in PubMed, CAS, Scopus and Google Scholar

- Research which is freely available for redistribution 